\title{
INJECTIVE VON NEUMANN ALGEBRAS
}

\author{
RICHARD I. LOEBL
}

\begin{abstract}
Injective von Neumann algebras are defined, and a characterization of them as complemented subspaces of $\mathscr{L}(H)$ is given. Several examples and applications are discussed.
\end{abstract}

The following observation shows an interesting relationship between the geometric structure of von Neumann algebras, i.e., the existence of projections of norm 1 onto them, and their extension properties as ranges of completely positive maps. Since this remark has some applications, e.g. [7], we wish to bring it to more widespread attention.

Following Stinespring [6], we say that a continuous linear map $\varphi$ between two $C^{*}$-algebras $\mathscr{A}$ and $\mathscr{B}$ is completely positive if $\forall n \geqq 1$, the natural map $\varphi_{n}$ from the $C^{*}$-algebra $\mathscr{A} \otimes M_{n}$ to the $C^{*}$-algebra $\mathscr{B} \otimes M_{n}$ is positive, where $M_{n}$ is the $C^{*}$-algebra of $n \times n$ matrices over the complex numbers.

We say that a von Neumann algebra $\mathscr{R}$ is injective if the following condition holds: for every $C^{*}$-algebra $\mathscr{B}$, for every selfadjoint linear subspace $S$ of $B$, containing the identity $e$ of $\mathscr{B}$, and for every completely positive linear map $\varphi: S \rightarrow \mathscr{R}$, there is a completely positive linear map $\psi: \mathscr{B} \rightarrow \mathscr{R}$ such that $\psi_{1_{\left.\right|_{s}}}=\varphi$.

Arveson [2] has shown that $\mathscr{L}(H)$, the von Neumann algebra of all bounded linear operators on a Hilbert space $H$, is injective; and further that, for any range algebra, such an extension is norm-preserving, since $\|\varphi\|=\|\varphi(e)\|=\left\|\varphi_{1}(e)\right\|=\left\|\varphi_{1}\right\|$.

Lemma. Let $\mathscr{R}$ be a von Neumann subalgebra of $\mathscr{L}(H)$, and let $P: \mathscr{L}(H) \rightarrow \mathscr{R}$ be such that $\|P\|=1, P_{\mid \mathscr{R}}=\mathrm{id}$; that is, $P$ is a Banach space projection of norm 1 . Then $P$ is completely positive.

Proof. The Lemma follows from Tomiyama [8].

THEOREM. A von Neumann subalgebra $\mathscr{R}$ of $\mathscr{L}(H)$ is injective iff there exists $P: \mathscr{L}(H) \rightarrow \mathscr{R}, P$ a Banach space projection of norm 1 .

Received by the editors March 16, 1973.

AMS (MOS) subject classifications (1970). Primary 46L10, 47D15; Secondary 46L05, 22D15.

Key words and phrases. Von Neumann algebras, injective, completely positive maps, property $P$, Banach space projection of norm one.

(c) American Mathematical Society 1974 
Proof. Suppose $\mathscr{R}$ is injective; then considering $\mathscr{R}$ as a norm-closed selfadjoint subspace containing the identity of $\mathscr{L}(H)$, the completely positive map id: $\mathscr{R} \rightarrow \mathscr{R}$ has an extension $P: \mathscr{L}(H) \rightarrow \mathscr{R}$. Clearly, $P$ is the desired projection.

Conversely, let $\mathscr{B}$ be any $C^{*}$-algebra, $S \subset \mathscr{B}$ a norm-closed selfadjoint subspace containing the identity of $\mathscr{B}$, and let $\varphi: S \rightarrow \mathscr{R}$ be a completely positive map. Let id: $\mathscr{R} \rightarrow \mathscr{L}(H)$ be the natural injection; then id is completely positive, and so is $\hat{\varphi}=\mathrm{id} \circ \varphi: S \rightarrow \mathscr{L}(H)$. By the results of Arveson [2], $\hat{\varphi}$ has a completely positive extension $\hat{\psi}: \mathscr{B} \rightarrow \mathscr{L}(H)$. Now, letting $P$ be a norm 1 projection onto $\mathscr{R}$, consider the map $\psi=P \circ \hat{\psi}$. By our lemma, $P$ is completely positive, hence so is $\psi$. Also, $\psi$ clearly extends $\varphi$. Q.E.D.

Remarks. (1) Hakeda and Tomiyama [4] say that a von Neumann algebra has the extension property if there exists a Banach space projection of norm 1 from $\mathscr{L}(H)$ onto $\mathscr{R}$. Hence, our result says that a von Neumann algebra is injective iff it has the extension property. It then follows from the work of Tomiyama [7] that $\mathscr{R}$ is injective iff $\mathscr{R}^{\prime}$ is.

(2) The reader may note the similarity between the idea of injective von Neumann algebras and $\mathscr{B}_{1}$-Banach spaces [3].

(3) We could have defined injective $C^{*}$-algebras, and it would follow as before that a $C^{*}$-algebra $\mathscr{A}$ is injective iff, considered as imbedded in $\mathscr{L}(H)$, there is a projection of norm 1 from $\mathscr{L}(H)$ onto $\mathscr{A}$. However, we feel that for applications the von Neumann algebra case is more important. One reason for this is a result of Akemann [1] that the only separable injective $C^{*}$-algebras (or von Neumann algebras) are finite dimensional, thus excluding most singly-generated operator algebras.

(4) It is a consequence of the results of Schwartz [5] that if a von Neumann algebra $\mathscr{R}$ has property $P$, then $\mathscr{R}^{\prime}$ is injective. Schwartz showed that every hyperfinite factor has property $P$. Combining this result with one of Tomiyama [7], we have

COROllary. Every hyperfinite factor is injective.

(5) Similarly, it follows from [4] and [7] that every Type I von Neumann algebra is injective. Hence the class of injective von Neumann algebras includes many interesting examples. However, by a modification of the argument in [5], it follows that there exists a factor of Type $\mathrm{II}_{1}$ which is not injective [7].

Question. Let $\mathscr{A}$ be an injective $C^{*}$-algebra. Is $\mathscr{A}^{\prime \prime}$ injective?

\section{BIBLIOGRAPHY}

1. C. Akemann, Projections onto separable $C^{*}$-subalgebras of a $W^{*}$-algebra, Bull. Amer. Math. Soc. 73 (1967), 925. MR 36 \#694. 
2. W. B. Arveson, Subalgebras of $C^{*}$-algebras, Acta Math. 123 (1969), 141-224. MR 40 \#6274.

3. M. Day, Normed linear spaces, Ergebnisse der Math. und ihrer Grenzgebiete, Heft 21, Springer-Verlag, Berlin, 1958. MR 20 \#1187.

4. J. Hakeda and J. Tomiyama, On some extension properties of von Neumann algebras, Tôhoku Math. J. (2) 19 (1967), 315-323. MR 36 \#5706.

5. J. Schwartz, Two finite, non-hyperfinite, non-isomorphic factors, Comm. Pure Appl. Math. 16 (1963), 19-26. MR 26 \#6812.

6. W. F. Stinespring, Positive functions on $C^{*}$-algebras, Proc. Amer. Math Soc. 6 (1955), 211-216. MR 16, 1033.

7. J. Tomiyama, The extension property of von Neumann algebras and a class of $C^{*}$-algebras associated to them, Københavns Univ. Mat. Inst. preprint series 1971, no. 3 .

8. - On the product projection of norm one in the direct product of operator algebras, Tôhoku Math. J. (2) 11 (1959), 305-313. MR 21 \#7453.

Department of Mathematics, University of California, Santa Cruz, California 95060

Current address: Department of Mathematics, Wayne State University, Detroit, Michigan 48202 